\title{
Extending SUSY reach at the CERN Large Hadron Collider using $b$-tagging
}

\author{
P. G. Mercadante, \\ Instituto de Física Teórica, Universidade Estadual Paulista, \\ São Paulo, Rua Pamplona 145, 01405-900, SP, Brazil \\ J. K. Mizukoshi, \\ Centro de Ciências Naturais e Humanas, Universidade Federal do ABC, \\ Rua Santa Adélia 166, 09210-170, Santo André, SP, Brazil \\ and Xerxes Tata \\ Department of Physics and Astronomy, University of Hawaii, Honolulu, HI 96822
}

\author{
Received on 20 November, 2006
}

\begin{abstract}
We analyze the potential of the CERN Large Hadron Collider on the reach of the focus point (FP) region in the mSUGRA parameter space. This region, consistent with WMAP results, is characterized by multi-TeV masses for the superpartners of quarks and leptons and relatively light charginos and neutralinos. Moreover, since the LSP has a substantial higgsino component, it is expected that the gluino decays predominantly to third generation quarks, producing a final state with multiple hard $b$ jets. Analyzing events with $\mathbb{E}_{T}+n$ jets + tagged $b$-jets, we show that the LHC reach can improve as much as $20 \%$ from current projections. Although we performed the analysis specifically for the FP region, the $b$-tagging should be important to enhance the SUSY signal in a variety of models where a relatively light gluino decays mostly to third generation quarks.
\end{abstract}

Keywords: SUSY; Supersymmetry; Supergravity; LHC; Jets

\section{INTRODUCTION}

Supersymmetry (SUSY) [1] is one of the most attractive models for new physics beyond the Standard Model. From the theoretical viewpoint one of the main motivation is that SUSY cures the quadratically divergent mass correction to the scalar particles. Moreover, in its minimum version, the minimal supersymmetric standard model (MSSM), the gauge couplings unify at the GUT scale.

The MSSM model, however, could contain more than a hundred free parameters due to the introduction of the soft SUSY breaking terms, namely masses, complex phases and mixing angles, leading to a model without predictive power. Nevertheles, if we make several assumptions, e.g. grandunification, we get highly predictive models, like the supergravity (mSUGRA) model [2]. In this specific model, SUSY is assumed to be dynamically broken in a "hidden sector" and communicated to a observable sector (with Standard Model particles and their superpartners) through gravitational interactions. The model is completely specified by just a few parameters, usually defined at some high scale (frequently taken to be $\left.\sim M_{G U T}\right)$. At this scale all scalar fields have a common SUSY breaking mass $m_{0}$, all gauginos have a mass $m_{1 / 2}$, and all soft SUSY breaking scalar trilinear couplings have a common value $A_{0}$. Electroweak symmetry breaking is assumed to occur radiatively. This fixes the magnitude of the superpotential parameter $\mu$. The soft SUSY breaking bilinear Higgs boson mass parameter $\left(B_{0}\right)$ can be eliminated in favor of $\tan \beta=v_{u} / v_{d}$ (the ratio of vacuum expectation value of Higgs fields), so that the model is completely specified by the parameter set:

$$
m_{0}, m_{1 / 2}, A_{0}, \tan \beta, \operatorname{sign}(\mu)
$$

In mSUGRA model $R$-parity is conserved, consequently the lightest SUSY particle (LSP), which is the lightest neutralino, is stable and therefore a good candidate for the cold dark matter (CDM). Recent measurements of the power spectrum of the cosmic microwave background (CMB) from the Wilkinson Microwave Anisotropy Probe (WMAP) and other experiments set the physical matter and baryon densities to be [3] $\Omega_{m} h^{2}=0.135_{-0.009}^{+0.008}$ and $\Omega_{b} h^{2}=0.0224 \pm 0.0009$, respectively, where $h$ is the Hubble constant in units of 100 $\mathrm{km} / \mathrm{s} / \mathrm{Mpc}$. The excess of non-baryonic matter results in $\Omega_{C D M} h^{2}=0.1126_{-0.009}^{+0.008}$. The upper limit derived from this is a true constraint on any stable relic from the Big Bang. Recently Baer et al. [4] mapped out the parameter space regions in the mSUGRA model preferred by CDM constraint, which can be probed at the LHC, and are consistent with indirect experimental constraints, namely rare decays $b \rightarrow s \gamma, b \rightarrow s \ell \bar{\ell}$, and $B_{s} \rightarrow \mu^{+} \mu^{-}$. Besides the canonical search $E_{T}^{\text {miss }}$ plus multijet and multilepton signal [5] at the LHC, the authors of Ref. [4] extended their analysis including the channels with isolated photon or with a leptonically decaying $Z$ boson.

In this talk we analyze one of region of parameter space favored by the CDM constraint, the so-called focus point (FP) region [6]. In Fig. 1, taken from Ref. [4], for a fixed value of $\tan \beta, \operatorname{sign}(\mu)$ and $A_{0}$, this region is a band just above the theoretically excluded region on the right-hand side. In this portion of parameter space the LSP has a substantial higgsino component, thus gluino and squarks decay predominantly into third generation quarks, leading to a final state with high $b$ jet multiplicity. In this scenario, an efficient $b$-tagging may improve substantially the discovery reach of supersymmetry over the canonical search, as we have shown in Ref. [7].

In the next section we show the main features of focus point, and in the section III we present our main results and 
conclusion. We encourage the reader to look at the Ref. [7] for further details and discussions.

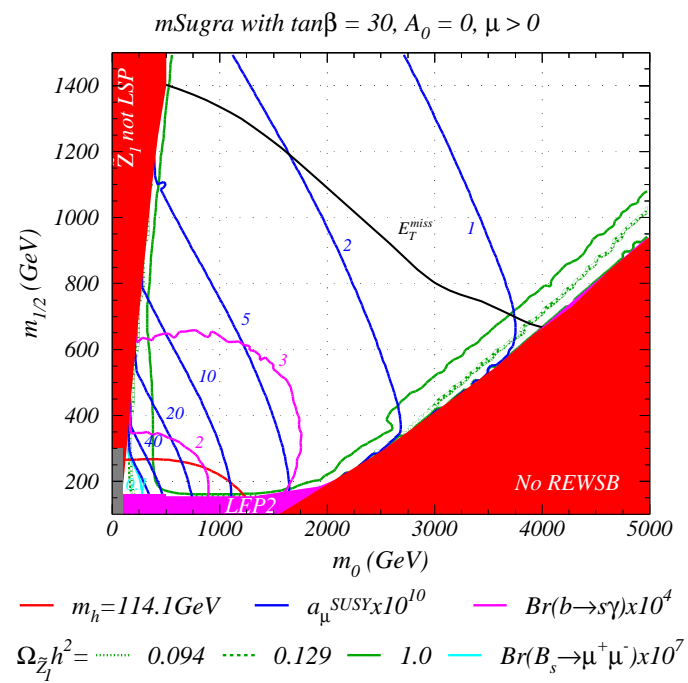

FIG. 1: Neutralino relic density constraint on the mSUGRA parameter space for $\tan \beta=30, A_{0}=0$ and $\mu>0$ along with LHC maximal reach and contours of several low energy observables obtained from Ref. [4].

\section{FOCUS POINT PHENOMENOLOGY AT THE LHC} by

In MSSM, the tree level $Z$ boson mass at weak scale given

$$
\frac{1}{2} m_{Z}^{2}=\frac{m_{H_{d}}^{2}-m_{H_{u}}^{2} \tan ^{2} \beta}{\tan ^{2} \beta-1}-\mu^{2} \sim-m_{H_{u}}^{2}-\mu^{2}
$$

is obtained by minimizing the Higgs potential. In particular the last relation holds for large $\tan \beta$. In this scenario naturalness criterion requires that there is no large cancellation between $m_{H_{u}}^{2}$ and $\mu^{2}$ when Eq. 2 is expressed in term of fundamental SUSY parameters. In mSUGRA the parameter set $\left\{a_{i}\right\}$ is given by Eq. 1, however for the naturalness discussion the authors of Ref. [6] consider the set $\left\{a_{i}\right\}=$ $\left\{m_{0}, m_{1 / 2}, \mu_{0}, A_{0}, B_{0}\right\}$ as the fundamental parameters.

The quantitative degree of fine-tuning of Eq. 2 is defined as

$$
c_{a_{i}} \equiv\left|\frac{\partial \ln m_{Z}^{2}}{\partial \ln a_{i}}\right| \text {. }
$$

In the Ref. [6] it was pointed out that a general class of supersymmetric theories, including mSUGRA, exhibits a focus point behavior in the MSSM renormalization Group Equations (RGEs). Fig. 2 shows this behavior for $m_{H_{u}}^{2}$, which starts from $m_{0}^{2}$ at GUT scale and assumes a negative value, providing the electroweak symmetry breaking at weak scale. Notice that no matter what value $m_{0}$ takes, there is a focus point at weak scale. It is important to mention that this behavior occurs when top quark assumes a value $m_{t} \sim 170-180 \mathrm{GeV}$, a range in agreement with its experimental value.

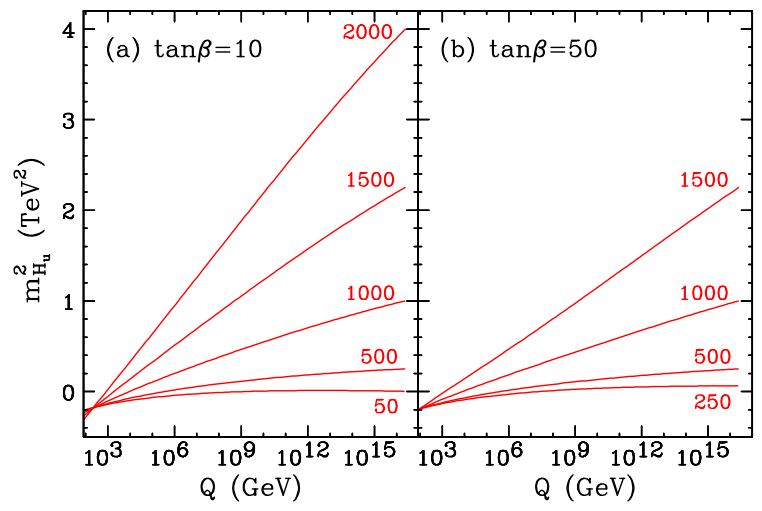

FIG. 2: The RG evolution for $m_{H_{u}}^{2}$ from Ref. [6]. In a) $\tan \beta=10$ and b) $\tan \beta=50$ for several values of $m_{0}$ (in units of $\mathrm{GeV}$ ), $m_{1 / 2}=300$ $\mathrm{GeV}, A_{0}=0$, and $m_{t}=174 \mathrm{GeV}$.

The focus point in the $m_{H_{u}}^{2}$ RGE implies that $c_{m_{0}}$ in Eq. 3 is small. Therefore the region of parameter space with $m_{0}$ larger than a few $\mathrm{TeV}$ is as natural as regions with $m_{0} \lesssim 1$ $\mathrm{TeV}$. Moreover, for large $\tan \beta, \mu$ becomes nearly a constant at weak scale and order of $\left|m_{H_{u}}\right| \sim m_{Z}$, provided that the higher order corrections do not spoil Eq. 2. In Fig. 1 this region is a band immersed in a portion of the parameter space favored by the CDM constraint, just above the theoretically excluded region on the right-hand side.

In the FP region $\widetilde{Z}_{1}, \widetilde{Z}_{2}, \widetilde{W}_{1}$ are higgsino like, therefore they are relatively light and they will be abundantly produced at the LHC. Since these particles are nearly degenerated, the decay product of $\widetilde{Z}_{2}$ and $\widetilde{W}_{1}$ will be rather soft leptons, which are difficult to be separated from the background. In this scenario, the SUSY signal at the LHC could come from gluino pair production through inclusive $E_{T}^{\text {miss }}$ channel. Indeed, as show in Fig. 1, for $100 \mathrm{fb}^{-1}$ of integrated luminosity, the LHC will be able to discover SUSY with gluino mass up to $m_{\tilde{g}} \sim 1.6-1.8 \mathrm{TeV}$.

In order to extend this reach, we observe that since the lighter neutralinos are higgsino-like, they couple more strongly with third generation than to first two generations. Therefore, decays of gluino into third generation fermions will be strongly enhanced so that the signal may be expected to be rich in high $E_{T} b$-jets [8].

For the multijet $+E_{T}^{\text {miss }}$ channel, the main SM background sources are $t \bar{t}$ production, $V+$ jet production $(V=W, Z)$ and QCD processes. Since the latter two backgrounds are not expected to be especially rich in hard bottom quark jets, and because experiments at the LHC are expected to have good $b$ tagging capability, we explore whether requiring the presence of tagged $b$-jets in the signal allows us to probe portions of the FP region that are inaccessible using the by now standard analyses [9-11] of the various multijet $+E_{T}^{\text {miss }}$ channels at the LHC. 
Rather than perform time consuming scans over the WMAP favored FP regions of the $m_{0}-m_{1 / 2}$ planes of the mSUGRA model for several values of $\tan \beta$, we have chosen three diverse model lines for our analysis. For each of these model lines, we take $\mu>0$ (the sign favored by the result of experiment E821 at Brookhaven [12]), $A_{0}=0$, and

- $m_{1 / 2}=0.295 m_{0}-477.5 \mathrm{GeV}$ with $\tan \beta=30$ for Model Line 1,

- $m_{1 / 2}=0.295 m_{0}-401.25 \mathrm{GeV}$ with $\tan \beta=30$ for Model Line 2, and

- $m_{1 / 2}=\frac{17}{60} m_{0}-390 \mathrm{GeV}$ with $\tan \beta=52$ for Model Line 3 .

For values of $m_{0} \gtrsim 1500 \mathrm{GeV}$, these model lines all lie in the WMAP allowed FP region of the mSUGRA parameter space delineated in Ref. [4]. The first two model lines have an intermediate value of $\tan \beta$ with Model Line 1 being deep in the FP region while Model Line 2 closer to the periphery of the corresponding WMAP region. We choose Model Line 3 again deep in the FP region, but with a very large value of $\tan \beta$ to examine any effects from a very large bottom quark Yukawa coupling. We take $m_{t}=175 \mathrm{GeV}$ throughout this analysis.

We have checked that for gluino with a mass $\sim 1650 \mathrm{GeV}$, which is close to the limit that can be probed at the LHC via the usual multijet + multilepton $+E_{T}^{\text {miss }}$ analyses, its branching ratio of decay into third generation is around $90 \%$, and around $35 \%$ of this ratio are due to the decay $\tilde{g} \rightarrow \widetilde{W}_{1}^{-} t \bar{b}+\widetilde{W}_{1}^{+} \bar{t} b$. Usually the decay patterns depend on both $\tan \beta$ and the value of $\mu / M_{1}$ ( i.e. on how deep we are in the HB/FP region), however the total branching fraction for decays to third generation quarks is relatively insensitive to these details. Motivated by these observations we begin our examination of the inclusive $b$-jet signal for each of the model lines introduced above.

\section{RESULTS AND CONCLUSION}

In our analysis we use the program ISAJET 7.69 [13] to simulate the signal and background events, and a toy calorimeter to reconstruct jets and leptons, as described in Ref. [9]. Jets are found using a cone algorithm with a cone size $\Delta R=\sqrt{\Delta \eta^{2}+\Delta \phi^{2}}=0.7$. Clusters with $E_{T}>40 \mathrm{GeV}$ and $\mid \eta($ jet $) \mid<3$ are defined to be jets. Muons (electrons) are classified as isolated if they have $E_{T}>10 \mathrm{GeV}(20 \mathrm{GeV})$ and visible activity in a cone with $\Delta R=0.3$ about the lepton direction smaller than $E_{T}<5 \mathrm{GeV}$. We identify a hadronic cluster with $E_{T} \geq 40 \mathrm{GeV}$ and $|\eta(j)|<1.5$ as a $b$ jet if it also has a $B$ hadron, with $p_{T}(B)>15 \mathrm{GeV}$ and $|\eta(B)|<3$, within a cone with $\Delta R=0.5$ of the jet axis. We take the tagging efficiency $\varepsilon_{b}=0.5$, and assume that gluon and other quark jets can be rejected as $b$ jets by a factor $R_{b}=150(50)$ if $E_{T}<100 \mathrm{GeV}$ $\left(E_{T}>250 \mathrm{GeV}\right)$ and a linear interpolation in between [14].

The major SM backgrounds to the channel $n$ jets $+E_{T}^{\text {miss }}$ signal come from $V+$ jet, $V=W^{ \pm}, Z, t \bar{t}$, and QCD productions of light quarks and gluons. In the last case, the $E_{T}^{\text {miss }}$ arises from neutrinos from $c$ and $b$ quarks, from showering of $W, Z$ bosons and their subsequent decays to neutrinos, and from energy mismeasurement.

In Fig. 3 we show the normalized cross sections for a typical signal, with gluino mass around $1700 \mathrm{GeV}$, and the background events as a function of the number of jets and $b$-jets. We have used the set of cuts

$$
\begin{aligned}
& N_{j} \geq 4, \\
& E_{T}^{\text {miss }}>400 \mathrm{GeV}, \\
& \left(E_{T}^{j 1}, E_{T}^{j 2}, E_{T}^{j 3}, E_{T}^{j 4}\right)>(400,250,150,100)(\mathrm{GeV}), \\
& m_{\mathrm{eff}}>1500 \mathrm{GeV},
\end{aligned}
$$

where $m_{\mathrm{eff}}$ is the scalar sum of the $E_{T}$ of the four hardest jets and $E_{T}^{\text {miss }}$, and we have obtained the following cross sections

$$
\begin{aligned}
\sigma(\text { signal }) & =1.1 \mathrm{fb}, \\
\sigma(t \bar{t}) & =5.17 \mathrm{fb}, \\
\sigma(W+\text { jets }) & =13.6 \mathrm{fb}, \\
\sigma(Z+\text { jets }) & =5.68 \mathrm{fb}, \\
\sigma(\mathrm{QCD}) & =27.7 \mathrm{fb} .
\end{aligned}
$$

For $m(\tilde{g})=1700 \mathrm{GeV}$, the cross section for the gluino pair production at the LHC is about $2.3 \mathrm{fb}$. This should be compared with $\sigma=1.1 \mathrm{fb}$ we get for the $n$ jets $+E_{T}^{\text {miss }}$ channel, with $n \geq 4$. If we take into account the reconstruction efficiencies, we see that the set of cuts above is rather soft for the signal, although it reduces significantly the SM backgrounds, which at this stage it will be dominated by $V+$ jets and QCD events. This is quite encouraging, since these processes usually have low $b$-jet multiplicity, if compared with the signal, as one can see from Fig. 3b). Besides kinematic variables above, there are others that can help to discriminate the signal from the backgrounds, namely $S_{T}$, the transverse sphericity, $\Delta \phi$, the azimuthal angle between $E_{T}^{\text {miss }}$ and the hardest jet, and $\Delta \phi_{b}$, the azimuthal opening angle between the two hardest $b$ jets in events with $N_{b} \geq 2$.

To quantify the improvement $b$-tagging makes to the capabilities of the LHC for the detection of SUSY, we have computed the reach of the LHC for each of the three model lines introduced above. Towards this end, for every mSUGRA parameter point that we examined, we generated a set of SUSY events using ISAJET. We also generated large samples of SM background events. We passed these events through the toy calorimeter simulation mentioned previously, and then analyzed both the signal and the background for the entire set of cuts $\left(5 \times 5 \times 6 \times 5 \times 3^{4}=60750\right.$ choices in all $)$, given by

i) $N_{j} \geq 4,5,6,7,8$;

ii) $E_{T}^{\text {miss }}(\mathrm{GeV})>400,500,600,700,800$;

iii) $\left(E_{T}^{j 1}, E_{T}^{j 2}, E_{T}^{j 3}, E_{T}^{j 4}\right)(\mathrm{GeV})>(400,250,150,100)$, $(400,250,175,125),(400,250,200,150),(500,350$, $150,100),(500,350,175,125),(500,350,200,150)$;

iv) $m_{\mathrm{eff}}(\mathrm{GeV})>\left[E_{T}^{\mathrm{miss}}+\sum_{i=1}^{4} E_{T}^{j i}\right]_{\min }+200 \times n, n=$ $0,1,2,3,4$;

v) $S_{T}>0.0,0.1,0.2$; 

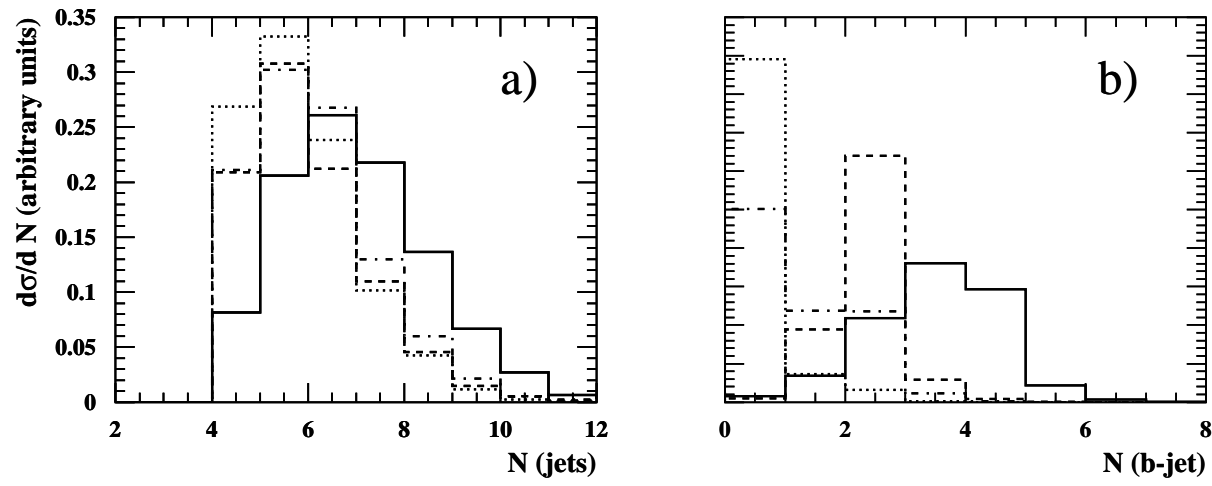

FIG. 3: Normalized cross section distributions for the signal (solid line), $t \bar{t}$ (dashed), $W$ and $Z+$ jets (dotted), and QCD (dash-dotted).

vi) $\Delta \phi<180^{\circ}, 160^{\circ}, 140^{\circ}$

vii) $\Delta \phi_{b}<180^{\circ}, 160^{\circ}, 140^{\circ}$;

viii) $N_{b} \geq 0,1,2$.

Notice that the cuts above are much harder than those used in analysis of the LHC SUSY signal [4]. This is because, unlike in Ref. [4] where the cuts were designed to extract the signal for a wide range of squark and gluino masses, here we focus on the optimization of the signal in the portion of the FP region with heavy gluinos where the previous strategy fails.

In our analysis, we consider a signal to be observable with a given integrated luminosity if,

a) statistical significance, $N_{S} / \sqrt{N_{B}} \geq 5$;

b) $N_{S} / N_{B} \geq 0.25$;

c) $N_{S} \geq 10$.

Our results for the LHC reach are shown in Fig. 4, where we plot the largest statistical significance of the signal as we run over the cuts i) to viii) for a) Model Line 1, b) Model Line 2, and c) Model Line 3. The solid curves, from lowest to highest, denote this maximum statistical significance without any $b$ tagging requirement, requiring $\geq 1$ tagged $b$ jet, and $\geq 2$ tagged $b$-jets respectively, for an integrated luminosity of $100 \mathrm{fb}^{-1}$, while the dotted curves show the corresponding results for $300 \mathrm{fb}^{-1}$ of integrated luminosity that may be expected from three years of LHC operation with the high design luminosity. While, without $b$-tagging, our reach for gluinos is $\sim 200 \mathrm{GeV}$ smaller than earlier projections $[4,10,11]$, we believe that the difference is due to the SM background estimation. While we use ISAJET, earlier works use the code PYTHIA, which does not include showering of $W$ and $Z$ bosons in QCD events. This difference, however, will not change the main conclusion of this work, that $b$-tagging will improve the mass reach of gluinos by $15-20 \%$, provided that LHC experiments can accumulate an integrated luminosity of $100-300 \mathrm{fb}^{-1}$ and that $b$-tagging with an efficiency of $\sim 50 \%$ remains possible even in the high luminosity environment.

A few remarks appear to be in order at this point:

- The statistical significance in Fig. 4 is not significantly improved if the $b$-tagging efficiency improves to $60 \%$. The reason is that before tagging the signal typically contains (on average) 3-4 $b$ quark jets while the background typically contains (at most) just two $b$ quark jets. As a result, the increased efficiency enhances the $b$-tagged background more than the signal, and the statistical significance is essentially unchanged.

- We have also checked that with a $b$-tagging efficiency of $50 \%$ and an integrated luminosity of $100 \mathrm{fb}^{-1}$, the signal with $\geq 3$ tagged $b$-jets is rate limited and no increase in the reach is obtained from that shown in the Figure.

- The search strategy proposed here does not use lepton information at all. We have checked that a transverse mass cut $M_{T}\left(\ell, E_{T}^{\text {miss }}\right) \gtrsim 100 \mathrm{GeV}$ on events with at least one isolated lepton does not increase the significance of the signal because the fraction of signal events (after our cuts) with an isolated lepton is not especially large.

- Although we have not shown this explicitly, we have checked that requiring the presence of additional isolated leptons does not lead to an increase in the reach relative to the $\geq 2 b$ channel.

In summary, we have shown that in the FP region of the mSUGRA model the reach of the LHC as measured in terms of gluino masses may be increased by $15-20 \%$ by requiring the presence of hard, tagged $b$-jets in SUSY events. While we were mainly motivated in our investigation by the fact that this part of parameter space is one of the regions compatible with 


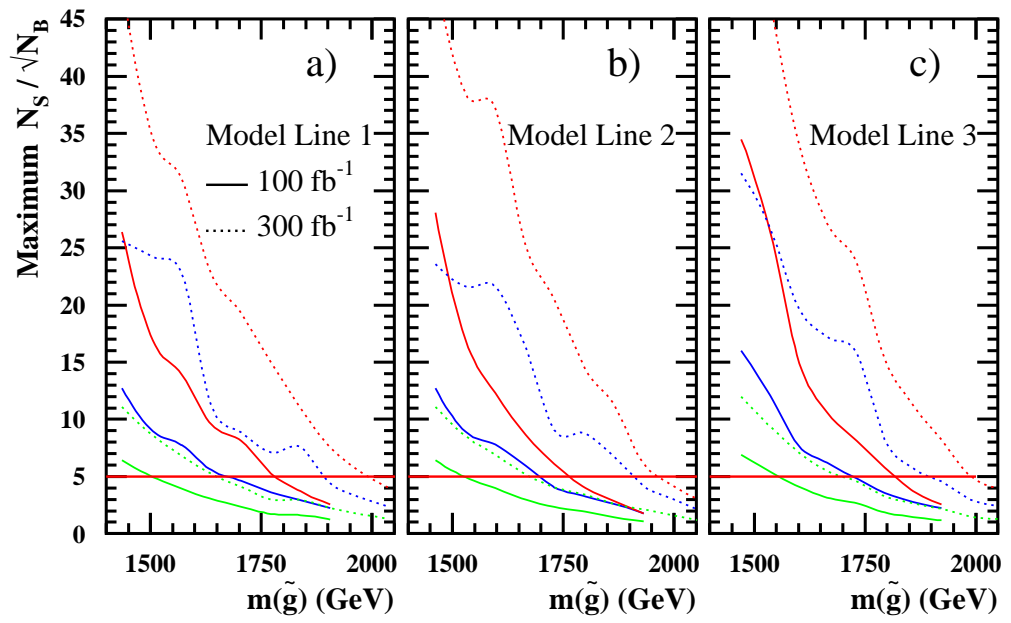

FIG. 4: The maximum value of the statistical significance for the multijet $+E_{T}^{\text {miss }}$ SUSY signal at the LHC as we run over the cuts i) to viii) for the three model lines introduced in the text, requiring in addition that $N_{S} \geq 10$ events and that $N_{S} \geq 0.25 N_{B}$. The solid lines from bottom to top denote this statistical significance without any $b$ tagging requirement, with at least one tagged $b$-jet, and with at least two tagged $b$-jets respectively, assuming an integrated luminosity of $100 \mathrm{fb}^{-1}$ and a tagging efficiency of $50 \%$. The dotted lines show the corresponding result for an integrated luminosity of $300 \mathrm{fb}^{-1}$.

the WMAP data, our considerations may have wider applicability. This is in part because the large top Yukawa coupling and, if $\tan \beta$ is large, also the bottom quark Yukawa coupling, cause the third generation squarks to be lighter than their siblings in the first two generations, and in part because of new contributions to gluino decay amplitudes from these large Yukawa couplings [15]. In particular, we may expect that $b$ tagging can be applied to models with an inverted squark mass hierarchy [16], in models with unification of Yukawa couplings, and possibly also in models with non-universal Higgs mass parameters that have recently been re-examined in light of the WMAP data [17].

Since the CMS and ATLAS experiments are expected to ultimately have good $b$-tagging capability, we urge that it be utilized to maximize the SUSY reach of the LHC.

\section{Acknowledgments}

This research was supported in part by the U. S. Department of Energy under contract number DE-FG-03-94ER40833 and by Fundação de Amparo à Pesquisa do Estado de São Paulo (FAPESP). JKM thanks Instituto de Física da Universidade de São Paulo, where part of research was conducted.
[1] For recent reviews, see e.g. S. Martin, in Perspectives on Supersymmetry, edited by G. Kane (World Scientific), hepph/9709356; M. Drees, hep-ph/9611409; J. Bagger, hepph/9604232; X. Tata, Proc. IX J. Swieca Summer School, J. Barata, A. Malbousson, and S. Novaes, Eds., hepph/9706307; S. Dawson, Proc. TASI 97, J. Bagger, Ed., hep$\mathrm{ph} / 9712464$; for a text book review of supersymmetry phenomenology, see M. Drees, R. Godbole and P. Roy, Theory and Phenomenology of Sparticles, (World Scientific, 2004).

[2] A. Chamseddine, R. Arnowitt, and P. Nath, Phys. Rev. Lett. 49, 970 (1982); R. Barbieri, S. Ferrara, and C. Savoy, Phys. Lett. B119, 343 (1982); L. J. Hall, J. Lykken, and S. Weinberg, Phys. Rev. D 27, 2359 (1983); for a review, see H. P. Nilles, Phys. Rep. 110, 1 (1984).
[3] C. L. Bennett et al., hep-ph/0302207; D. N. Spergel et al., hep$\mathrm{ph} / 0302209$.

[4] H. Baer et al., JHEP06, 054 (2003).

[5] H. Baer, C-h. Chen, F. Paige, and X. Tata, Phys. Rev. D 52, 2746 (1995); ibid. 53, 6241 (1996).

[6] J. L. Feng, K. T. Matchev, and T. Moroi, Phys. Rev. Lett. 84, 2322 (2000); Phys. Rev. D 61, 075005 (2000); hep-ph/0003138.

[7] P. G. Mercadante, J. K. Mizukoshi, and X. Tata, Phys. Rev. D 72, 035009 (2005).

[8] U. Chattopadhyay, A. Datta, A. Datta, A. Datta, and D. P. Roy, Phys. Lett. B 493, 127 (2000).

[9] H. Baer, C-H. Chen, F. Paige, and X. Tata, Phys. Rev. D 52 , 2746 (1995); 56, 6241 (1996); H. Baer, C-H. Chen, M. Drees, F. Paige, and X. Tata, Phys. Rev. D 59, 055014 (1999). 
[10] ATLAS Collaboration, Technical Design Report, CERN LHCC/99-15 (1999); B. Allanach, J. Hetherington, A. Parker, and B. Webber, JHEP 08, 017 (2000).

[11] S. Abdullin and F. Charles, Nucl. Phys. B547, 60 (1999); S. Abdullin et al. (CMS Collaboration), hep-ph/9806366.

[12] G. Bennett et al. (E821 Collaboration), Phys. Rev. Lett. 89, 101804 (2002); 92, 161802 (2004).

[13] F. Paige, S. Protopopescu, H. Baer, and X. Tata, hep$\mathrm{ph} / 0312045$.

[14] S. Corréad, V. Kostioukhine, J. Levêque, A. Rozanov, and
J. B. de Vivie, ATLAS Note, ATLAS-PHYS-2004-006, and V. Kostioukhine, ATLAS Note, ATLAS-PHYS-2003-033.

[15] H. Baer, C-H. Chen, M. Drees, F. Paige, and X. Tata, Phys. Rev. Lett. 79, 986 (1997); 80, 642 (1998) (E).

[16] See e.g. H. Baer et al., Phys. Rev. D 64, 015002 (2001), and references therein.

[17] H. Baer, A. Mustafayev, S. Profumo, A. Belyaev, and X. Tata, Phys. Rev. D 71, 095008 (2005), and hep-ph/0504001, JHEP (in press). 